\title{
Probabilistic Monte-Carlo Method for Modelling and Prediction of Electronics Component Life
}

\author{
T. Sreenuch, A. Alghassi and S. Perinpanayagam \\ Integrated Vehicle Health Management Centre \\ Cranfield University \\ Bedford MK43 0AL, UK
}

\author{
Y. Xie \\ Shanghai Aircraft Design and Research Institute \\ Commercial Aircraft Cooperation of China \\ Shanghai 201210, P. R. China
}

\begin{abstract}
Power electronics are widely used in electric vehicles, railway locomotive and new generation aircrafts. Reliability of these components directly affect the reliability and performance of these vehicular platforms. In recent years, several research work about reliability, failure mode and aging analysis have been extensively carried out. There is a need for an efficient algorithm able to predict the life of power electronics component. In this paper, a probabilistic Monte-Carlo framework is developed and applied to predict remaining useful life of a component. Probability distributions are used to model the component's degradation process. The modelling parameters are learned using Maximum Likelihood Estimation. The prognostic is carried out by the mean of simulation in this paper. Monte-Carlo simulation is used to propagate multiple possible degradation paths based on the current health state of the component. The remaining useful life and confident bounds are calculated by estimating mean, median and percentile descriptive statistics of the simulated degradation paths. Results from different probabilistic models are compared and their prognostic performances are evaluated.
\end{abstract}

Keywords-Prognostics; Monte-Carlo Simulation; Remaining Useful Life

\section{INTRODUCTION}

Nowadays in order to provide warning and predict failures to avoid catastrophic failure of products and systems, there has been an increasing tendency in monitoring the ongoing "health" of them [1]. The insulated gate bipolar transistor (IGBT) modules play an increasing significant role in on-wing for avionics system, such as communication system in autonomous working, radar system and navigation system and so on. The failures of IGBT components can degrade the efficiency of the systems or result in system failures [1]. In general, IGBT modules have several thousand hours' lifetime expectancy [2], but in order to analyze failures from several of them, the lifetime of the modules needs to be reduced. Therefore the process that causes it to fail must still operate the module within its specifications, but in a greatly reduced time frame.

IGBT accelerated aging system is to design and implement a system capable of performing robust experiments on gate controlled power transistors to induce and analyze prognostic indicators [3]. The main goal for the development of experiment system was to identify precursor parameters for device failure. Precursor parameters are parameters of the device that change with time wherein the change can be mapped to degradation in the device. Once the precursor parameters are identified, suitable diagnostic and prognostic algorithms can be implemented using these parameters to provide early warning of failure and predict remaining useful life [4].

Hence, a comprehensive approach to the development of a prognostic framework for IGBTs is required, there is a necessity to develop methods to predict the remaining useful life (RUL) of IGBTs to prevent system stoppage and costly failures. Prognostic is a technology under ongoing development. The technology aims towards high technology sectors, for example the automotive or aerospace industries, for ensuring safety and customer satisfaction. Most modern vehicles monitor their systems to ensure correct operation. If a fault is detected or predicted the user of the vehicle is usually notified before the fault has had a detrimental effect on the vehicle. Modern vehicles also monitor their usage and change their service intervals accordingly. The reliability of IGBTs directly affect the reliability and performance of these vehicle system. In recent years, series of research work about IGBT reliability, failure mode and aging analysis has been carried out widely, and a suitable prognostic method for IGBT and an efficient algorithm for predicting the IGBT RUL become increasingly important.

As electronic components have an increasingly consumption in new generation aircrafts and vehicles, and the amount of electronic failure will also become significant. Fault diagnosis and prognostic, estimation of remaining useful life and health management have vital roles to avoid catastrophic failure, improve aircraft reliability, reduce maintenance cost and increase performance [5]. This paper bases its study on IGBT for development of algorithms for estimating remaining useful life of components, and it is considered to contribute to the prognostic technology development in integrated vehicle health management (IVHM) field and advance the electronic components prognosis.

There are several approaches that have been developed for electronic prognostics. The issues unaddressed in previous IGBT prognostics studies will form the basis for the motivation of the current study. [6] used a system model approach to estimate the remaining useful life of lithium ion batteries. The battery was represented by a lumped parameter model. The parameters of the model were calculated using relevance vector machine (RVM) regression on experimental data. An extended Kalman filter and particle filter algorithms were used to determine the battery RUL.

[7] describes the use of prognostic cells to predict failure in integrated circuits. The prognostic cell was developed to fail 
prior to the circuit on the same chip for all realistic operating conditions. Prognostic monitors in the test cell experienced the exact environment that the actual circuit experienced, but at an accelerated rate, thereby providing failure prediction. [8] used the data-driven approach to detect anomalies of notebook computers by monitoring performance parameters and comparing them against the historical data using Mahalanobis distance.

A physics-based prognostic approach was used by [7] in the development of a diagnostic system based on a virtual system. Using a Virtual Test Bed (VTB), system faults found in a real world system were simulated along with a normally operating real world system. For the development of a fault diagnostic system for a brake-by-wire system, [9] used a similar fuzzy system approach. Six failure modes of the system were identified and three measurement points chosen. The input signals were processed on a segment-by-segment basis, by a feature extraction process, and by fault detection.

The aim of this paper is to develop a prognostic approach that is applicable to power electronic components and computational efficient embeddable in a low power device. Here, IGBT is used a case study. The outline of this paper is as follows: Section I describes the background of the prognostic is described and summarizes the current research work on IGBT. Section II describes IGBT accelerated aging experiments, IGBT aging data. The aging data are processed and the degradation profiles of IGBT are analyzed. In section III, the maximum likelihood method is utilized to computing the parameters of IGBT degradation models. In section IV, Monte Carlo simulation method and IGBT degradation models are used to predict the RUL, and the algorithm of IGBT prognostic is developed. The RUL prediction results are analyzed in section $\mathrm{V}$ and the error and root mean square error are analyzed to compare the efficiency of different models in predicting the RUL. Section VI concludes the paper and the future works are discussed.

\section{IGBT DEGRADATION PROFILE}

\section{A. Aging Experiments}

The IGBT accelerated aging experiments are designed to study the aging characters of the IGBT and develop the algorithm of prognostic for prediction of the remaining useful life. The IGBT degradation data set is acquired from the aging process system, which is provided by the AMES laboratory of NASA [10]. The data set can be used to design and develop prognostic algorithms for semiconductor components such as IGBTs which have increasingly been used in modern multiple vehicle systems. IGBT accelerated aging experiments belong to the project in NASA to investigate the degradation characterizations of electronic components [11], as electronic components have an increasingly consumption in new generation aircrafts and vehicles, and the amount of electronic failure will also become significant. Fault diagnosis and prognostic, estimation of remaining useful life and health management have a vital role to avoid catastrophic failure, improve aircraft reliability, reduce maintenance cost and increase performance.
IGBT accelerated aging experiments are based on the aging platform which induces the degradation and electronic faults into the test system. Prevalently, four kinds of accelerate aging methods are widely used in accelerated aging experiments, which are thermal cycling, hot carrier injection, electrical over stress and time dependent dielectric breakdown stimulus [12]. The IGBT functional failure such as die solder degradation and wire lift were brought by the thermal cycling accelerate aging approach. Hot carrier injection could accelerate electrons and holes pass into gate oxide, which could result in the increase of IGBT threshold voltage. IGBT condition mutation and lighting could be caused by the electrical overstress due to the excessive voltage, current or power. The breakdown of IGBT gate oxide will happen when the charge injection exceeds the threshold which is caused by accumulating of the temperature in the gate oxide when it is being operated [12]. Accelerated aging approaches such as thermal cycling and electrical overstress are used in IGBT accelerate aging experiments to speed up the degradation and failure of the IGBT in experiments environments which simulate the scenarios of industrial practical application. Precursor parameters, such as collector voltages, collector currents, gate voltages and currents, and environmental parameters such as temperature are monitored and recorded to be utilized for IGBT diagnosis and prognosis research [13].

The experiment data and measurements are shared in the website of NASA as an open database which can be used to develop prognostic algorithms available to academic and industrial researchers [10]. IGBT accelerated aging data set are measurements and sensor data collected from IGBT accelerated aging experiments platform shown in figure 1 . The data set includes the measurements being recorded from IGBT experiments (or operating) environment and survey data representing the deterioration of IGBT in the experiments. This data set contains mass data from thermal overstress aging experiments, including several parameters being recorded continuously such as collector current, collector voltage, gate voltage, package temperature etc. [14]. These data and parameters were monitored and recorded constantly until the IGBT failure in accelerate aging experiments. The data set were formatted in a data array which could be read by MATLAB to facilitate analysis and processing for the data in the subsequent research and investigation.

Figure 2 depicts a process of the prognostic algorithm development used in this paper. Firstly, IGBTs are tested in accelerated aging experiments following standard experiment procedures in an environmental simulation scenario to accelerate aging and failure. The monitoring data and experiments parameters are recorded and collected to transport into the software platform which are used as data formation and data storage. IGBT diagnostic and prognostic investigation will based on these data set. Prognostic algorithm for RUL prediction will be developed, and Monte Carlo simulation is used in the prognostic algorithm which will be described in more detail in the subsequent sections. 


\section{B. Degradation Data}

The aim of data processing is to gain useful information from the data with the approach of analysis and sorting. Collector emitter voltage is selected as a precursor parameter for the IGBT aging prognostic in this paper [15]. The profile of the $\mathrm{V}_{\mathrm{CE}}$ collected from the aging experiment is presented in figure 3 . The collector emitter voltage of the IGBT presents a monotone increasing in the whole aging process and the $\mathrm{V}_{\mathrm{CE}}$ is also presents a fluctuation and oscillation during this process, but the $\mathrm{V}_{\mathrm{CE}}$ falls quickly at the end of the aging process when the IGBT becomes to fail. The whole aging process is more than 10000 time units. The whole aging process is more than 10000 time units.

The aging data of raw $\mathrm{V}_{\mathrm{CE}}$ as a precursor parameter are processed by low-pass filtering, and its filtered profile is shown in figure 4 . It can be seen that $\mathrm{V}_{\mathrm{CE}}$ presents an increase step by step during the whole IGBT aging process. The data is now clean and more suitable for the analysis. The variation of $\mathrm{V}_{\mathrm{CE}}$ in the whole aging process could be separated into 7 stages, and the values of $\mathrm{V}_{\mathrm{CE}}$ for each phase are discretely different. Seven IGBTs were used in the accelerated aging experiment. It can be seen that the degradation stages are clearly separated from each other. The time duration where $\mathrm{V}_{\mathrm{CE}}$ stays in each stage are computed and listed in table I. The $\mathrm{V}_{\mathrm{CE}}$ voltage value is approximately $2.45 \mathrm{~V}$ at the starting of the aging process. The degradation states can be determined by

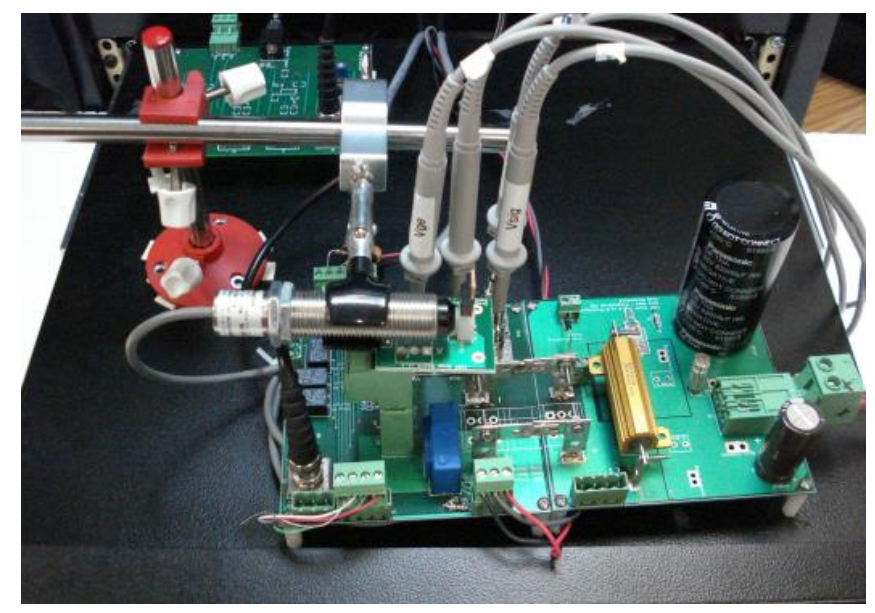

Fig. 1. IGBT accelerated aging experiments hardware [10].

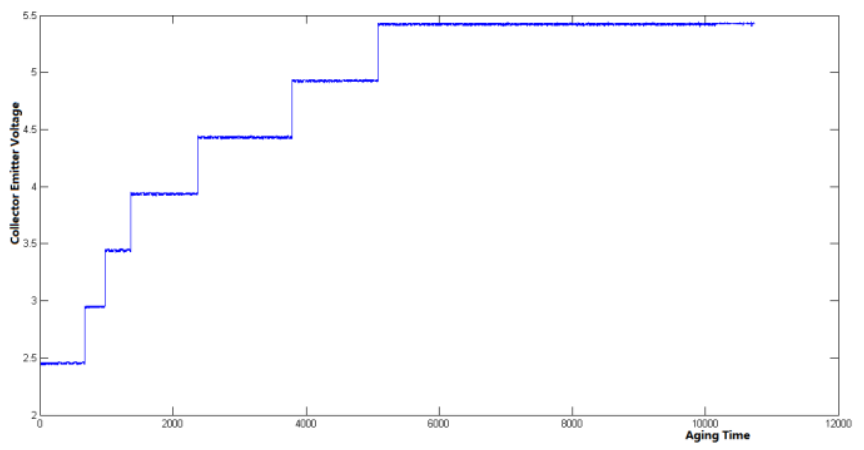

Fig. 4. Collector-Emitter Voltage after K-Mean Clustering.

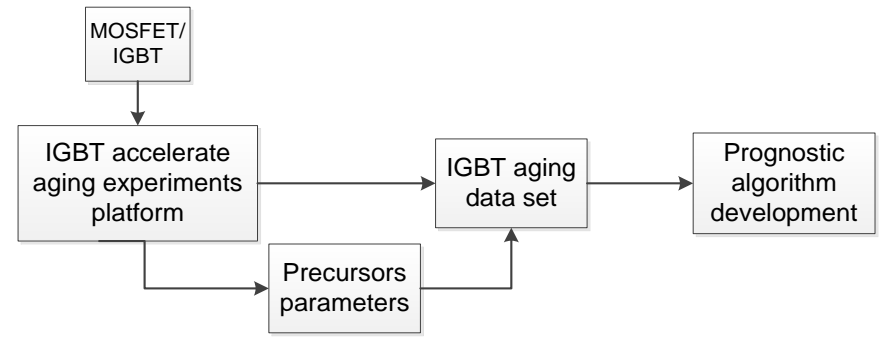

Fig. 2. Process of IGBT prognostic algorithm development.

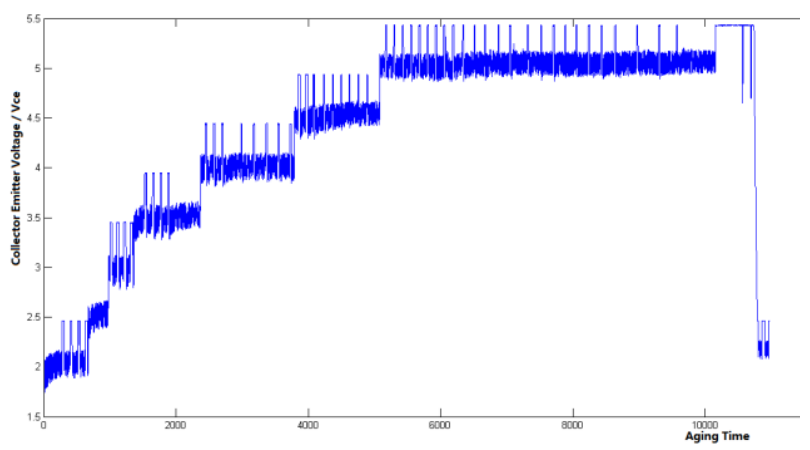

Fig. 3. Collector-Emitter Voltage Profile.

the level of $\mathrm{V}_{\mathrm{CE}}$. In this particular IGBT, $\mathrm{V}_{\mathrm{CE}}$ increases about $0.5 \mathrm{~V}$ discrete step at each degradation phase.

TABLE I. IGBT DEGRADATION DATA SETS.

\begin{tabular}{|c|c|c|c|c|c|c|c|}
\hline & \multicolumn{7}{|c|}{ IGBT degradation process } \\
\hline $\begin{array}{c}\text { IGBT } \\
\text { No. }\end{array}$ & $\begin{array}{c}\text { 1st } \\
\text { Phase }\end{array}$ & $\begin{array}{c}\text { 2nd } \\
\text { Phase }\end{array}$ & $\begin{array}{c}\text { 3rd } \\
\text { Phase }\end{array}$ & $\begin{array}{c}\text { 4th } \\
\text { Phase }\end{array}$ & $\begin{array}{c}5 \text { th } \\
\text { Phase }\end{array}$ & $\begin{array}{c}\text { 6th } \\
\text { Phase }\end{array}$ & Failure \\
\hline 1 & 670 & 970 & 1368 & 2369 & 3793 & 5079 & 10740 \\
\hline 2 & 827 & 827 & 1389 & 1389 & 2306 & 3208 & 5075 \\
\hline 3 & 1099 & 1099 & 1905 & 2490 & 2900 & 3889 & 6799 \\
\hline 4 & 894 & 894 & 1733 & 2384 & 2789 & 3887 & 5141 \\
\hline 5 & 927 & 1055 & 2115 & 2544 & 3388 & 7449 & 10285 \\
\hline 6 & 578 & 578 & 1560 & 2109 & 3403 & 4236 & 12164 \\
\hline 7 & 750 & 1631 & 2755 & 3001 & 3757 & 5079 & 6861 \\
\hline
\end{tabular}

\section{MAXIMUM LIKELIHOOD ESTIMATION}

\section{A. Degradation Model}

Table II records the run-to-failure degradation process of 7 IGBT samples used in the accelerated aging experiment. The columns are the time durations of each degradation phases. It can be seen that an IGBT will degrade and undergo 6 degradation phases before it eventually fail. Each phase will last for a period of time before the degradation progresses further to the next phase. Take the first IGBT for example, the operational use life of IGBT-No.1 is 5079 unit time, and the duration of the IGBT stayed in its first degeneration phase is 
670 unit time, then the IGBT degraded into the second degradation phase and stayed 300 unit time before its further degradation to step into the third degradation phase. And so on, until the IGBT had stayed for 1286 unit time in the last phase, the IGBT continued degraded and completely failed.

In this paper, the occurrence of degradation (or time duration of each degradation phase) is assumed to be random and uncorrelated to other degradation phases. Therefore, 6 independent stochastic process models could be built to represent the degradation phases which follow the random probability distribution. In this paper, Gamma, Exponential and Poisson distributions are used in modeling the degradation process.

TABLE II. IGBT DEGRADATION PHASE DURATION.

\begin{tabular}{|c|c|c|c|c|c|c|c|}
\hline & \multicolumn{7}{|c|}{ Duration of Each Phase } \\
\hline $\begin{array}{c}\text { IGBT } \\
\text { No. }\end{array}$ & $\begin{array}{c}\text { 1st } \\
\text { Phase }\end{array}$ & $\begin{array}{c}\text { 2nd } \\
\text { Phase }\end{array}$ & $\begin{array}{c}\text { 3rd } \\
\text { Phase }\end{array}$ & $\begin{array}{c}\text { 4th } \\
\text { Phase }\end{array}$ & $\begin{array}{c}5 \text { th } \\
\text { Phase }\end{array}$ & $\begin{array}{c}\text { 6th } \\
\text { Phase }\end{array}$ & $\begin{array}{c}\text { IGBT } \\
\text { Life }\end{array}$ \\
\hline 1 & 670 & 300 & 398 & 1001 & 1424 & 1286 & 5079 \\
\hline 2 & 827 & 0 & 562 & 0 & 917 & 902 & 3208 \\
\hline 3 & 1099 & 0 & 806 & 585 & 410 & 989 & 3889 \\
\hline 4 & 894 & 0 & 839 & 651 & 405 & 1098 & 3887 \\
\hline 5 & 927 & 128 & 1060 & 429 & 844 & 4061 & 7449 \\
\hline 6 & 578 & 0 & 982 & 549 & 1294 & 833 & 4236 \\
\hline 7 & 750 & 881 & 1124 & 246 & 756 & 1322 & 5079 \\
\hline
\end{tabular}

The IGBT degradation and failure are considered to be random, and hence the duration time $\left(\mathrm{T}_{\mathrm{i}}\right)$ of the degeneration phase is considered to be a random variable, see figure 5 . The $y$-axis in the figure represents the collector emitter voltage of the IGBT, and the $\mathrm{x}$-axis represents the aging time of the experiment. The figure indicates that the duration time $\left(T_{i}\right)$ in which the IGBT was measured in different volt of $\mathrm{V}_{\mathrm{CE}}$ is a random variable and it could be represented using the probability density functions summarized in table III.

\section{B. Modelling Parameters}

In this paper, the Maximum Likelihood method is used to estimate the parameters listed in table III for the Gamma, Exponential and Poisson distribution models. In order to estimate the model parameters, the duration time of 7 IGBTs in degradation process are used as statistical samples, and the six degradation phases are considered to be uncorrelated stochastic processes.

For the Gamma distribution, there are two modelling parameters $\kappa$ and $\theta$ to be estimated. It is assumed that the duration of each degradation phase are uncorrelated and follow the Gamma probability distribution defined in table III. Maximum Likelihood Estimation (MLE) is generically formulated as

$$
\mathrm{L}(\kappa, \theta)=\prod_{i=1}^{n} f\left(x_{i}, \kappa, \theta\right)
$$

TABLE III. DISTRIBUTION FUNCTIONS AND MODELLING PARAMETERS [16].

\begin{tabular}{|c|c|c|}
\hline Models & Density Functions & Parameters \\
\hline Gamma & $\mathrm{f}\left(T_{i}=\mathrm{x}\right)=x^{k-1}\left(\mathrm{e}^{\frac{-\mathrm{x}}{\theta}} / \Gamma(\mathrm{k}) \theta^{\mathrm{k}}\right)$ & $\kappa, \theta$ \\
\hline Exponential & $\mathrm{f}\left(T_{i}=\mathrm{x}\right)=\lambda e^{-\lambda x}$ & $\lambda$ \\
\hline Poisson & $\mathrm{f}\left(T_{i}=\mathrm{x}\right)=\frac{e^{-\lambda} \lambda^{k}}{k !}$ & $\lambda$ \\
\hline
\end{tabular}

from which $\kappa$ and $\theta$ can be analytically estimated using [17]

$$
\begin{gathered}
\hat{\theta}=\frac{1}{\kappa N} \sum_{i=1}^{N} x_{i} \\
\hat{\kappa} \leftarrow \kappa-\frac{\ln (\kappa)-\psi(\kappa)-s}{\frac{1}{\kappa}-\psi^{\prime}(\kappa)}
\end{gathered}
$$

Table IV summarizes the parameters for 6 uncorrelated degradation phases obtained from MLE.

TABLE IV. MLE FOR GAMMA PROBABILITY DistRIBUTION.

\begin{tabular}{|c|c|c|c|c|c|c|}
\hline Parameters & Model 1 & Model 2 & Model 3 & Model 4 & Model 5 & Model 6 \\
\hline K & 25.77 & 0.0883 & 9.3663 & 0.2818 & 5.1662 & 3.3289 \\
\hline$\theta$ & 31.8 & 211.67 & 88 & 1754.4 & 167.3 & 450.2 \\
\hline
\end{tabular}

There is only one modelling parameter $\lambda$ for the Exponential distribution model. Similar to Gamma distribution, the duration of each degradation phase are assumed to be uncorrelated. In this paper, $\lambda$ can be estimated using an analytical MLE solution [17]

$$
\hat{\lambda}=\frac{1}{\bar{x}}=\frac{n}{\sum_{i=1}^{n} x_{i}}
$$

and the estimated parameters are listed in table $\mathrm{V}$.

TABLE V. MLE FOR EXPONENTIAL PROBABILITY DISTRIBUTION.

\begin{tabular}{|c|c|c|c|c|c|c|}
\hline Parameter & Model 1 & Model 2 & Model 3 & Model 4 & Model 5 & Model 6 \\
\hline$\lambda$ & 820.7 & 187 & 824.4 & 494.4 & 864.3 & 1498.7 \\
\hline
\end{tabular}




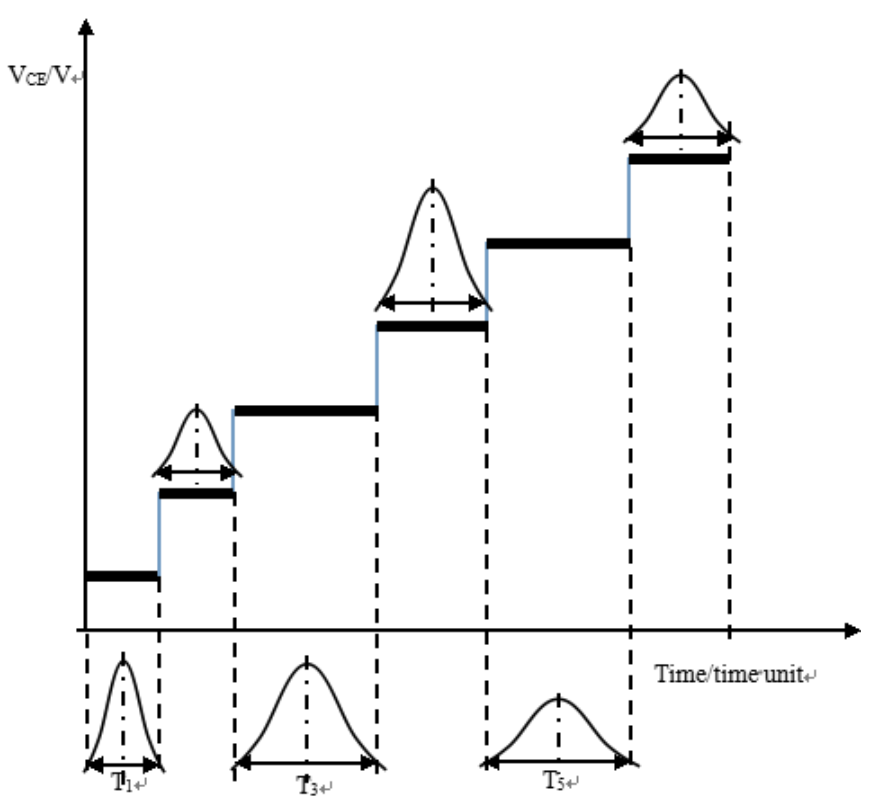

FIGURE 5: IGBT Degradation Model.

For Poisson probability distribution, similar to Exponential, $\lambda$ is only the modelling parameter that needs to be estimated. $\lambda$ can be analytically derived, and its MLE can be calculated using [17]

$$
\hat{\lambda}=\frac{1}{\bar{x}}=\frac{n}{\sum_{i=1}^{n} x_{i}}
$$

Table VI summarizes the parameters for 6 uncorrelated degradation phases obtained from MLE.

TABLE VI. MLE FOR POISSON PROBABILITY DISTRIBUTION.

\begin{tabular}{|c|c|c|c|c|c|c|}
\hline Parameter & Model 1 & Model 2 & Model 3 & Model 4 & Model 5 & Model 6 \\
\hline$\lambda$ & 820.7 & 187 & 824.4 & 494.4 & 864.3 & 1498.7 \\
\hline
\end{tabular}

\section{PROGNOSTIC APPROACH}

In section III, the IGBT degradation models have been developed based on the probabilistic distributions and tuned using the data obtained from the accelerated aging experiments. Based on the degradation profiles shown in figure 3 and 4 , the degradation process can be observed by tracking the $\mathrm{V}_{\mathrm{CE}}$ measurement values. The profile indicates that $\mathrm{V}_{\mathrm{CE}}$ monotonically increases in discrete steps. In this paper, Monte Carlo simulation is utilized to generate the degradation paths to represent the time durations the IGBT stays in different degradation phases.

Figure 6 shows a block diagram of the prognostic algorithm developed in this paper. The aging data sets were used to train the degradation model depending on what probability distributions are employed. In this paper, the RUL of the IGBT component is predicted by the mean of simulation. The Monte Carlo simulation is used to propagate degradation paths into the future. The RUL can either be the mean or median RUL of the multiple propagated paths. The $\mathrm{V}_{\mathrm{CE}}$ measurements provide regular updates to the determination or confirmation of the current degradation phase. For every measurement updates, the Monte Carlo simulation is re-run to generate new degradation paths based on the updated measurement and the RUL is then re-calculated from the newly generated paths.

Figure 7 shows an example of IGBT degradation paths. It is assumed that the duration $T_{i}$ follows Gamma, Exponential or Poisson probability distributions. $S_{\mathrm{i}}$ is the ending time of the degradation phase of the sequence $i$. $t$ is the elapse time started from the beginning of the experiment. 6 stochastic models are built based on the 6 degradation phases, where the related MLE parameters are summarized in table IV-VI. The RUL prediction could be calculated using the equation

$$
R U L_{P}=S_{f}-t
$$

where $\mathrm{RUL}_{P}$ means the predicted RUL by either Gamma, Exponential or Poisson models, and $\mathrm{S}_{\mathrm{f}}$ is the predicted IGBT failure time in the aging process. $S_{\mathrm{f}}$ is also the ending time of the last degeneration phase. The precursor parameter $\mathrm{V}_{\mathrm{CE}}$ is used to determine in which degradation phase the IGBT is. When the IGBT is stay in the first degeneration phase, then:

$$
S_{f}=\sum_{i=1}^{6} T_{i}
$$

where $T_{i}$ is generated by Monte Carlo simulation and it means the duration time of relevant phase. So the predicted RUL could be represented as:

$$
R U L_{P}=S_{f}-t=\sum_{i=1}^{6} T_{i}-t
$$

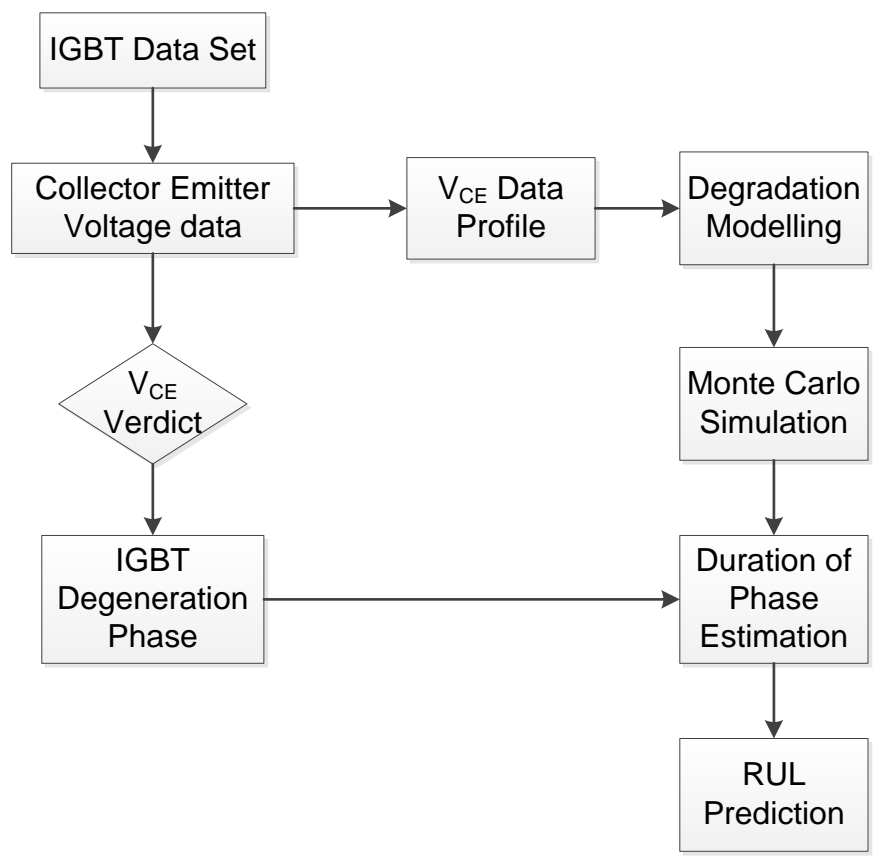

Fig. 6. RUL Prediction Process. 


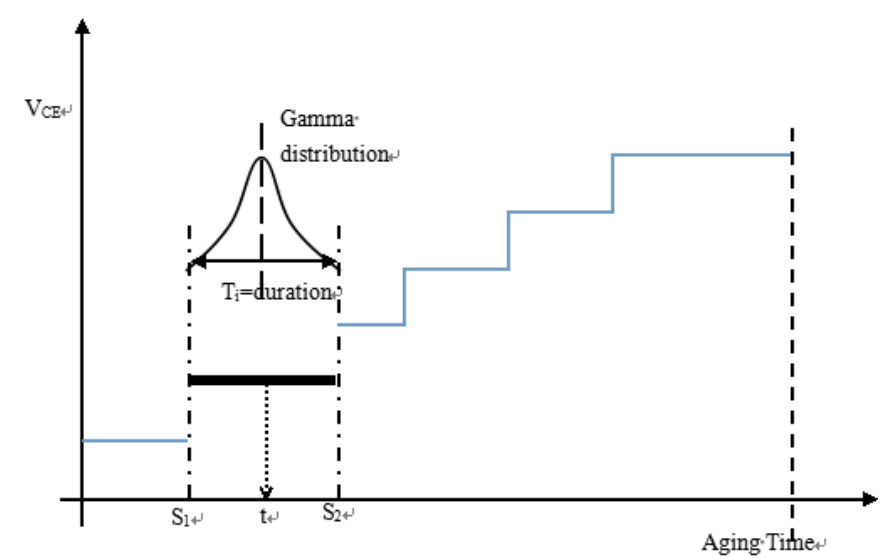

Fig. 7. Probability Model for RUL Prediction.

When the IGBT stays in the ordinal of $\mathrm{i}$ degeneration phase, because the past generation phase is monitored by measurements which is the $V_{C E}$. So $S_{i-1}$ is a known number could be surveyed from the $\mathrm{V}_{\mathrm{CE}}$ data recording. Then the predicted RUL could be calculated as:

$$
R U L_{P_{i}}=S_{f}-t=\left(S_{f}-S_{i-1}\right)-\left(t-S_{i-1}\right)
$$

Then

$$
R U L_{P_{i}}=\sum_{i}^{6} T_{i}-\left(t-S_{i-1}\right)
$$

Where if the simulated $\mathrm{Ti}$ generated by Monte Carlo simulation is larger than $\left(\mathrm{t}-\mathrm{S}_{\mathrm{i}-1}\right)$, it means that the component is still and will be continue in this degradation phase, or the component has ended the generation phase and begin to jump

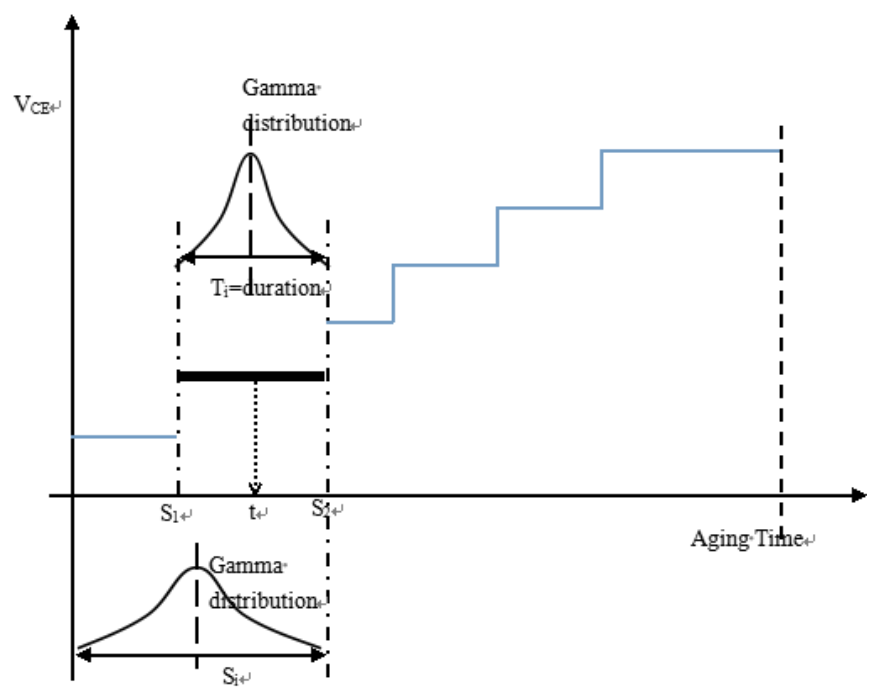

Fig. 8. Combined Model for RUL Prediction. into the next degradation phase.

Note that Monte Carlo simulation is used to generate the duration time of each phase $\left(T_{i}\right)$. In order to improve the accuracy of prediction, a large number of multiple runs is needed, which is 500 in this paper. The RUL prediction result uses the mean value and the median value of the distribution.

A combination of statistical properties can be also used to improve prognostic accuracies. One can be based on the duration time of the degradation phase, and the other can be based on the ending time of the degradation phase. Figure 8 illustrates the use of a combined model for predicting the RUL. prediction. The distribution of these two kind of probability models are combined to predict the IGBT RUL. When the IGBT stays in the ordinal of i degeneration phase, the ending time of the phase is $S_{i}$, and $S_{i}$ could be represented as:

$$
S_{i}=S_{i-1}+T_{i}
$$

Where $T_{i}$ is the duration time of this degeneration phase which will be generated by Monte Carlo simulation, and $S_{i-1}$ is the ending time of the prior degradation phase. So $S_{i-1}$ is a known number. $T_{i}$ is simulated by Monte Carlo. It follows the model distribution based on the duration time of the degradation phase. The probability of $S_{\mathrm{i}}$ is different from the distribution of $T_{i}$, which means two probability distribution for $\mathrm{S}_{\mathrm{i}}$ has been established. Combining these two distributions to predict the RUL is the main solution in this model.

\section{RESUlts}

\section{A. RUL Predictions}

The IGBT RUL prediction results are expressed by a series of polylines. The RUL prediction is a continue process from the beginning of the IGBT running to the end of the process when IGBT is becoming failed. The sensor data are recorded at each moment of the IGBT degradation process and the RUL prediction are also carried out at each moment of the degradation process. Hence, the RUL prediction happens through the whole process of the IGBT degradation experiment.

Figure 9 shows an example (i.e. IGBT sample number 1) of RUL prognostic results. The result was computed based on Gamma distribution model. The straight blue, green and yellow dash lines are used as the real and $\pm 10 \%$ deviation RUL, respectively. They are used as baselines to indicate how well the prognostic algorithm performs during the test. In figure 9, the red and blue scatter plots are the mean and median values of the RUL prediction. The green and yellow plots are the 90 and 10 percentiles of the Monte Carlo simulated degradation paths. At the beginning of the rendering test, the RUL prediction is lower than the real RUL value, however as the predicted RUL slowly converges to the real value as the operating time is towards the end of component life. 


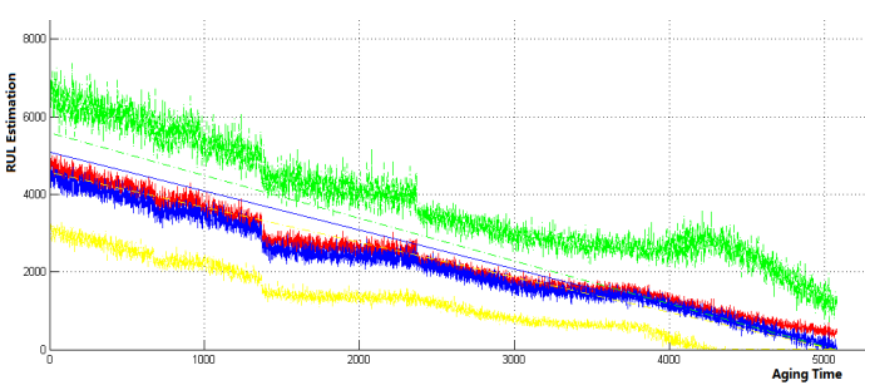

Fig. 9. Example of RUL Prediction Results using Gamma Distribution.

Figure 10 shows an example (i.e. IGBT sample number 1) of RUL prognostic results based on Exponential distribution model. The red, blue green and yellow scatter plots are the mean, median, 90 and 10 percentiles of the simulated degradation paths. Similar to Gamma distribution model, the predicted RUL slowly converges to the real RUL value as the $\mathrm{V}_{\mathrm{CE}}$ measurements are rendered towards the end of component life. However, the 10 and 90 percentile bounds are significantly different from the results obtained in the Gamma distribution case. The width of these bounds is too wide and practically become meaningless information-wise in this case. In Exponential distribution, $\lambda$, i.e. mean, is the only parameter in the model. This model lacks of additional parameter that represents the statistical description equivalent to the standard deviation. This explains the practically irrelevant of the 10 and 90 percentile bounds if these values to be derived based on the Exponential distribution model.

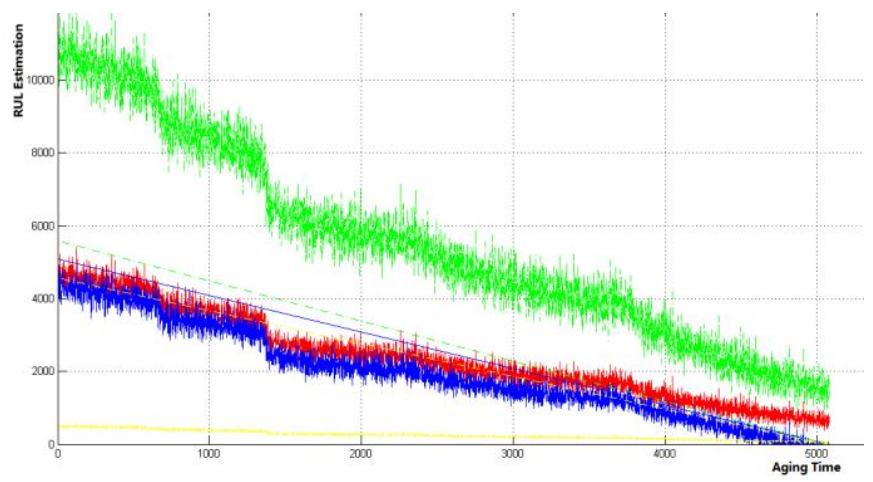

Fig. 10. Example of RUL Prediction Results using Exponential Distribution

An example (i.e. IGBT sample number 1) of Poisson based RUL prognostics results is shown in figure 11. Similarly to Gamma and Exponential results, the predicted RUL slowly converges to the real RUL value as the operating cycles close to the end of component life. The degradation paths are linear with sudden changes reflected the discrete change in the degradation state updated from the $\mathrm{V}_{\mathrm{CE}}$ measurement. In contrast to Exponential distribution, the 10 and 90 percentile scatter plots lie very close to the mean and median values. These bounds are unrealistic close and practically do not provide meaningful information in terms of confident in the RUL prediction.

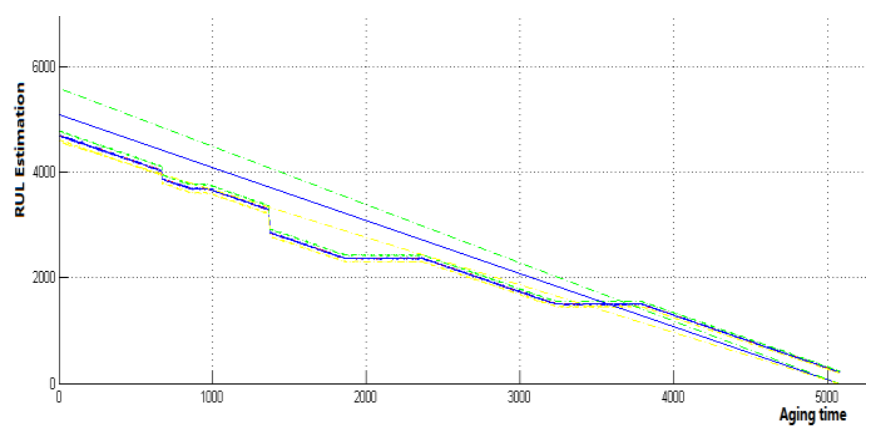

Fig. 11. Example of RUL Prediction Results using Poisson Distribution.

Figure 12 shows an example (i.e. IGBT sample number 1) of RUL prognostic results based on the combined (Gamma) distribution model. The combined model gives the prognostic results, i.e. mean and median, as accurate as the Gamma model. However, the 10 and 90 percentiles are much tighter than the results obtained from the Gamma distribution. In this case, these confident bounds lie close to the $\pm 10 \%$ deviation of the real RUL. Hence, the 10 and 90 percentile bounds calculated using the combined model practically provides more meaningful confident intervals in comparison to the Gamma, Exponential and Poisson distribution models.

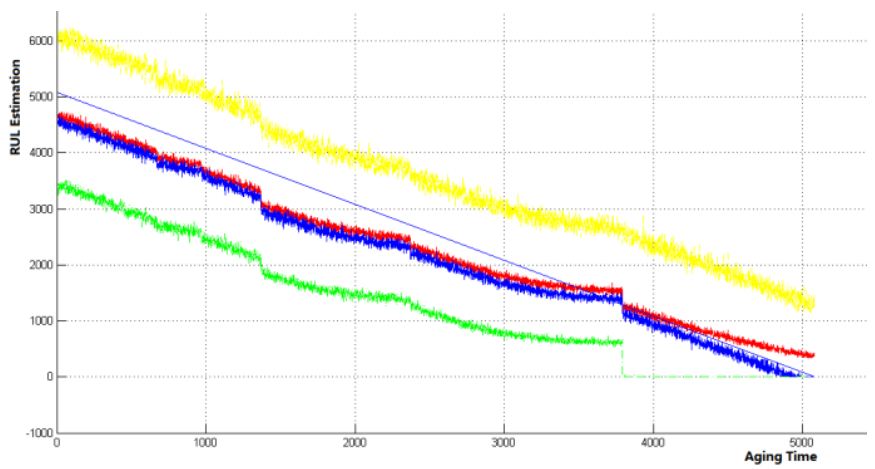

Fig. 12. Example of RUL Prediction Results using Combined Model

\section{B. Error Analysis}

The errors between the predicted and real RUL values reflect the performance of the IGBT prognostic approach. In this paper, the prediction (or prognostic) error is defined by

$$
E_{r}=R U L_{R}-R U L_{P}
$$

where $E_{\mathrm{r}}$ is the error value between the predicted and real values, RUL $L_{R}$ is the real RUL value of an IGBT and RUL ${ }_{P}$ is the predicted value obtained from the prognostic algorithm. Using equation (12), the prognostic accuracy can be quantitatively calculated using

$$
P_{p r}=\frac{E_{r}}{R U L_{R}}=\frac{R U L_{R}-R U L_{P}}{R U L_{R}}
$$


In this paper, root mean square error (RMSE) is used to measure the prognostic performance of different probability distribution models. The RMSE can be calculated using the following equations:

$$
\begin{gathered}
\text { MSE }=\frac{1}{n} \sum_{i=1}^{n}\left(\widehat{Y}_{l}-Y_{i}\right)^{2} \\
\mathrm{MSE}=\frac{1}{T_{f}} \sum_{i=1}^{T_{f}}\left(R U L_{P_{i}}-R U L_{R_{i}}\right)^{2}=\frac{1}{T_{f}} \sum_{i=1}^{T_{f}}\left(E r_{i}\right)^{2} \\
\mathrm{RMS}=\sqrt{\frac{1}{n} \sum_{i=1}^{n}\left(\widehat{Y}_{l}-Y_{i}\right)^{2}}=\sqrt{\frac{1}{T_{f}} \sum_{i=1}^{T_{f}}\left(E r_{i}\right)^{2}}
\end{gathered}
$$

Figure 13 and 14 summarize the mean and median based RMSEs of different probability distribution models tested against 7 IGBT data samples. For the mean based RUL, the Poisson model performs better than other models on most of the test samples; Its RMSE is less than other models. However, for the median based RUL, the Exponential model has less RMSE values in comparison to other models. Comparing the RMSE of mean and median predicted RULs, the IGBT test sample number 1 and 7 have the smallest RMSE for combining model. The rest of other IGBT test samples have similar prognostic performance in terms of how different probabilistic models performed in relation to each other.

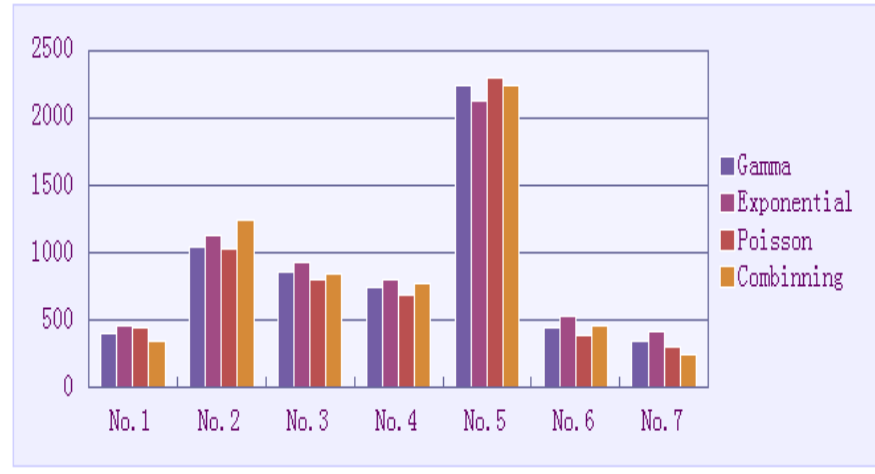

Fig. 13. RMSE for Mean Based of Predicted RULs.

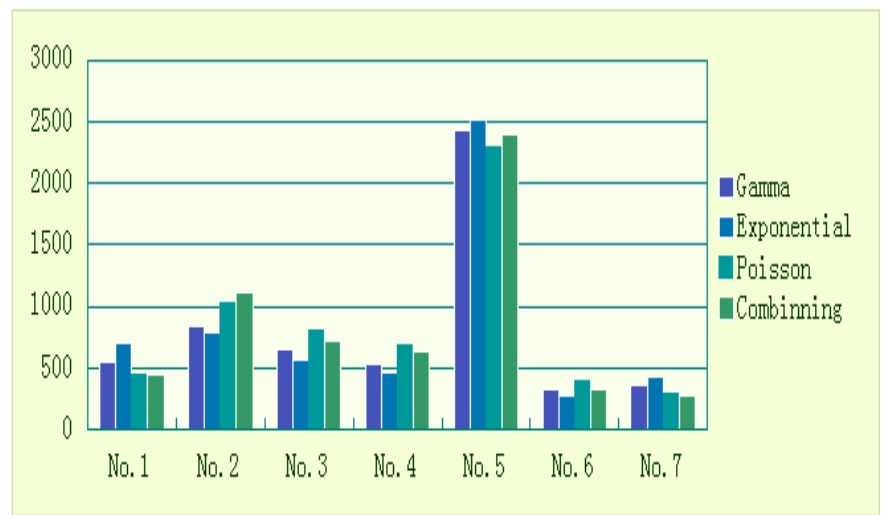

Fig. 14. RMSE for Median Based of Predicted RULs.

\section{CONCLUSIONS}

The main contribution of this paper is the development and implementation of a prognostics framework for IGBTs, and a prognostic algorithm with Monte Carlo simulation and with the collector emitter voltage as a precursor parameter is developed. According to the IGBT failure mechanism and degradation characterization, the IGBT degeneration models are built. Monte Carlo simulation method and the precursor parameter, collector emitter voltage $\left(\mathrm{V}_{\mathrm{CE}}\right)$, are integrated to develop the prognostic algorithm on predicting the IGBT RUL.

Gamma, Exponential, Poisson distribution and the combining distribution models are established, and Monte Carlo simulation is utilized in the algorithm to computing the IGBT remaining useful life. The collector emitter voltage $\left(\mathrm{V}_{\mathrm{CE}}\right)$ is used as the precursor parameter used in the prognosis.

Comparing with the results of RUL prediction with different models, the mean value of the RUL prediction and the median value of the RUL prediction presents a different accuracy. Different models also perform their preference to different IGBT on the RUL prediction. The implementation of the developed prognostics framework could be applied to provide advance warning of failures thereby preventing costly power electronics system downtime and failures.

The combining model can perform much more efficient RUL prediction results in some IGBTs, and the combined model in this paper is only based on the Gamma distribution, much more combining models based on different probability distribution could be established and implemented in IGBT RUL prediction, and a comparative analysis between these combining models is beneficial to the IGBT prognostic. 


\section{References}

[1] J. Celaya, B. Saha and P. Wysocki, "Prognostics for Electronics Components of Avionics Systems," in IEEE Aerospace Conference, Big Sky, MT, 2009.

[2] J. Celaya, A. Saxena, C. Kulkarni, S. Saha and K. Goebel, "Prognostics Approach for Power MOSFET under Thermal-Stress Aging," in Reliability and Maintainability Symposium (RAMS), Reno, NV, 2012.

[3] M. Pecht and R. Jaai, "A prognostics and health management roadmap for information and electronics-rich systems," Microelectronics Reliability, pp. 317-323, 2010.

[4] N. Patil, D. Das, K. Goebel and M. Pecht, "Identification of Failure Precursor Parameters for Insulated Gate Bipolar Transistors (IGBTs)," in INTERNATIONAL CONFERENCE ON PROGNOSTICS AND HEALTH MANAGEMENT, Denver, CO, 2008.

[5] B. Saha, J. Celaya, P. Wysocki and K. Goebel, "Towards Prognostics for Electronics Componenets," Aerospace conference, IEEE, no. 2009, pp. $1-7,2009$

[6] B. Saha, K. Goebel and S. Poll, "Modeling Li-ion Battery Capacity Depletion in a Particle Filtering Framework," in Annual Conference of the Prognostics and Health Management Society, San Diego, 2009.

[7] M. Pecht, Prognostics and Health Management of Electronics, New York, NY: Wiley-Interscience, 2008.

[8] N. Patil, D. Das and M. Pecht, "Aprognostic approach for non-punch through and field stop IGBTs," Special section on International Seminar on Power Semiconductors 2010, vol. 52, no. 3, pp. 482-488, 2010.
[9] Y. Murphey, A. Masur and B. Chen, "A Fuzzy System for Fault Dignostics in Power Electronics Based Brake-by-wire System," in Annual Meeting of the North American Fuzzy Information Processing Society, 2005, New York.

[10] NASA, "NASA," 24 June 2013. [Online]. Available: http://ti.arc.nasa.gov/tech/dash/diagnostics-and-prognostics.

[11] J. Celaya, P. Wysocki, V. Vashchenko and S. Saha, "Accelerated Aging System for Prognotics of Power Seminconductor Devices," in Autotestcon, 2010 IEEE, Orlando, FL, 2010.

[12] G. Sonnenfeld, K. Goebel and J. Celaya, "An Agile Accelerated Aging, Characterization and Scenario Simulation System for Gate Controlled Power Transistors," in IEEE AUTOTESTCON, Salt Lake City, UT, 2008.

[13] N. Patil, D. Das, K. Goebel and M. Pecht, "Failure Precursors for Insulated Gate Bipolar Transistors (IGBTs)," IEEE TRANSACTIONS ON RELIABILITY, pp. 271 - 276, 2009.

[14] J. Celaya, P. Wysocki and K. Goebel, "IGBT accelerated aging data set," NASA Ames Prognostics Data Repository, Moffett Field, CA, 2009.

[15] A. Alghassi, S. Perinpanayagam and I. Jennions, "A Simple State-Based Prognostic Model for Predicting Remaining Useful," in Power Electronics and Applications (EPE), 2013 15th European Conference, Lille, France, 2013.

[16] J. Lawless, Statistical Model and Methods for Lifetime Data, John Wiley \& Sons, 2011.

[17] R. B. Millar, Maximum Likelihood Estimation and Inference, John Wiley \& Sons, 2011. 